\title{
Palmitate induces CD11b expression in monocytes independent of altered redox state
}

\author{
C. Pararasa, C. J. Bailey and H. R. Griffiths \\ Life and Health Sciences, Aston University, Aston Triangle, Birmingham B4 7ET, UK
}

Ageing has been associated with increased oxidative stress ${ }^{(1)}$ and elevated risk of developing CVD $^{(2)}$ and insulin resistance ${ }^{(3)}$. Studies in middle-aged individuals suggest that elevated serum saturated fatty acids are indicative of greater CVD and type-2 diabetes risk ${ }^{(4)}$. Furthermore, serum SFA levels increase with age, whereas levels of the cellular antioxidant glutathione decline ${ }^{(5)}$. Whether age-associated changes to SFA levels contribute to or arise from an ageing metabolic phenotype, altered redox state and/or vascular inflammation is unknown.

This work has determined the effect of the SFA, palmitate, on intracellular redox status and expression of the integrin CD11b in THP-1 monocytes, a cell surface marker that mediates monocyte interaction with the endothelium.

THP-1 cells were incubated with 50,150 and $300 \mu \mathrm{M}$ palmitate conjugated to albumin and albumin-vehicle control as previously described $^{(6)}$. This was not associated with a significant loss of viability. The total intracellular level of glutathione (GSH+GSSG) was measured using a DTNB-dependent recycling assay and corrected for protein content determined by BCA assay ${ }^{(7)}$. Monocyte CD11b expression was determined using flow cytometry ${ }^{(8)}$.

Palmitate treatment $(24 \mathrm{~h})$ depleted total glutathione $(\mathrm{GSH})$ levels in THP-1 monocytes: this was significant at $50 \mu \mathrm{M}$, but at higher concentrations of palmitate, the GSH levels were not significantly different to control values (Fig. 1). CD11b expression increased dose dependently with increasing palmitate concentration, confirming previous observations ${ }^{(6)}$.

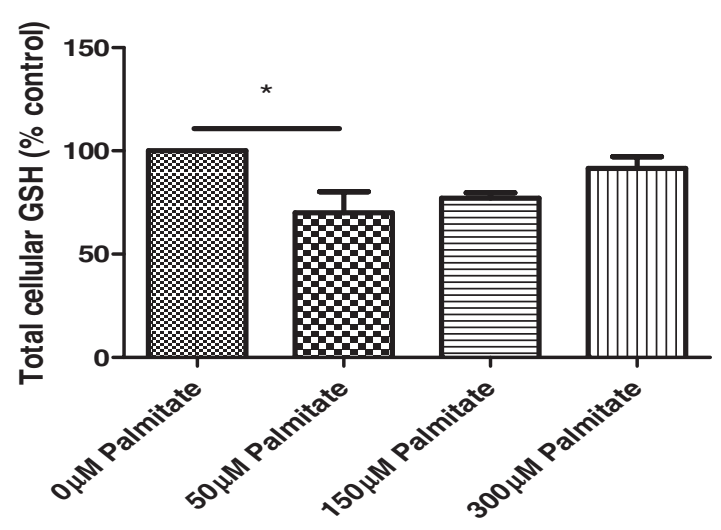

Fig. 1. Monocyte incubation with palmitate for $24 \mathrm{~h}$ depletes cellular glutathione. $* P<0.05 v$. control.

The change to cellular glutathione levels indicates that the incubation of monocytes with $50 \mu \mathrm{m}$ palmitate induces a redox shift after $24 \mathrm{~h}$ that is not evident at higher palmitate concentrations. However, the redox shift did not correlate with the enhanced CD11b expression observed with increasing palmitate concentrations.

Together, these data suggest that palmitate may mediate the increased CD11b expression leading to a pro-inflammatory monocyte phenotype typically seen in older adults and manifest as an increased binding of monocytes to endothelium. However, CD11b expression changes are not associated with a redox shift, suggesting that the two phenomena are unrelated.

1. Chung HY et al. (2009) Molecular inflammation: Underpinnings of aging and age-related diseases. Ageing Res Rev 8, 18-30.

2. Fares E \& Howlett SE (2010) Effect of age on cardiac excitation-contraction coupling. Clin Exp Pharmacol Physiol. 37, 1-7.

3. Fink RI et al. (1983) Mechanisms of insulin resistance in aging. J Clin Invest 71, 1523-1535.

4. Wang L et al. (2003) Plasma fatty acid composition and incidence of coronary heart disease in middle aged adults: the atherosclerosis risk in communities (ARIC) study. Nutr Metab Cardiovasc Dis 13, 256-266.

5. Rebrin I \& Sohal RS (2008) Pro-oxidant shift in glutathione redox state during aging. Adv Drug Deliv Rev 60, $1545-1552$.

6. Gao D, Griffiths HR \& Bailey CJ (2007) Palmitate induces insulin resistance in monocytes and increases expression of the integrin CD11b. Proc Nutr Soc 66, 44A.

7. Grant MM, Barber VS \& Griffiths HR (2005) The presence of ascorbate induces expression of brain derived neurotrophic factor in SH-SY5Y neuroblastorna cells after peroxide insult, which is associated with increased survival. Proteomics 5, 534-540.

8. Woollard KJ, Phillips DC \& Griffiths HR (2002) Direct modulatory effect of C-reactive protein on primary human monocyte adhesion to human endothelial cells. Clin Exp Immunol 130, 256-262. 